\title{
The WHAT Project
}

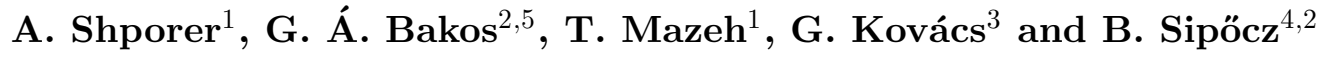 \\ ${ }^{1}$ Wise Observatory, Tel Aviv University, Tel Aviv 69978, Israel \\ email: shporer@wise.tau.ac.il \\ ${ }^{2}$ Harvard-Smithsonian Center for Astrophysics (CfA), 60 Garden Street, Cambridge, MA \\ 02138, USA \\ ${ }^{3}$ Konkoly Observatory, Budapest, P.O. Box 67, H-1125, Hungary \\ ${ }^{4}$ Department of Astronomy, Eötvös Loránd University, Pf. 32, H-1518 Budapest, Hungary \\ ${ }^{5} \mathrm{NSF}$ fellow
}

\begin{abstract}
WHAT is a small-aperture short focal length automated telescope with an $8.2^{\circ} \times 8.2^{\circ}$ field of view, located at the Wise Observatory, Israel. The system is similar to the HATNet telescopes $\dagger$ and is aimed at searching for transiting extrasolar planets and variable objects. Operational since 2004, WHAT has accumulated $\sim 100000$ exposures of several fields and was part of the discovery of the transiting planet HD147506b. Further description of WHAT can be found at: http://wise-obs.tau.ac.il/ what.
\end{abstract}

WHAT is a Wise observatory Hungarian-made Automated Telescope, located at the Wise Observatory in Mizpe Ramon, Israel. It is operated under a collaboration between the Wise Observatory of the Tel-Aviv University and Konkoly Observatory of the Hungarian Academy of Sciences. Similar to other HAT telescopes (Bakos et al. 2002, 2004), WHAT is a combination of a fully automated telescope mount, clamshell dome (to protect the telescope), $2 \mathrm{~K} \times 2 \mathrm{~K}$ (4 megapixel) CCD, $200 \mathrm{~mm}$ f/1.8 Telephoto lens, PC running RealTime Linux and a software environment (ProMount) controlling all the above.

WHAT operation is fully automated. The only manual interaction is that of establishing weather conditions and enabling the telescope on each evening. This nightly monitoring is done by a teamwork from Wise and Konkoly Observatories.

WHAT monitors the intensity of many thousands of stars in large fields of views, $8.2^{\circ} \times 8.2^{\circ}$ each, searching for transiting planets and variable stars. The position of WHAT at a longitude of $34^{\circ} 46^{\prime}$ E makes the HATNet-WHAT an around-the-globe network. WHAT is located 9.7 hours in longitude from the four HAT instruments at FLWO, Arizona, and 11.3 hours from the two HAT instruments at Mauna Kea, Hawaii. A wide span around the globe is important for continuous observations of the HATNet fields, increasing the number of observed transit events.

Since it has become operational in 2004 WHAT has accumulated some 100000 exposures of several fields, most of which were observed in collaboration with other HATNet instruments. Table 1 lists the amount of data obtained by WHAT for fields where a total of more than 4000 HATNet-WHAT observations have already been done.

Analysis of the data and identification of transiting planet candidates, showing a transit-like signal, is usually done independently in Konkoly, CfA and Tel Aviv. The detected candidates are debated within the group and those found worthy are passed on for spectroscopic follow-up observations. Figure 1 shows one of our candidates, HTR177004, with a clear transit-like signal and a magnitude drop of only 3 mmag. Although

$\dagger$ http://cfa-www.harvard.edu/ gbakos/HAT/, see Bakos et al. in this volume. 
Table 1. List of HATNet fields where WHAT obtained a significant fraction of the observations, and a total of over 4000 observations were done by HATNet-WHAT. The left-most column lists the HATNet field number, the middle column lists the number of WHAT observations and total amount of HATNet-WHAT observations, and the right-most column gives the collaborating HATNet telescope ID.

\begin{tabular}{lll}
\hline $\begin{array}{l}\text { HATNet Field } \\
\text { Number }\end{array}$ & WHAT /Total & $\begin{array}{l}\text { Collaborating } \\
\text { Telescope }\end{array}$ \\
\hline 211 & $1486 / 4429$ & HAT-8 \\
210 & $3088 / 6169$ & HAT-6 \\
212 & $4873 / 4873$ & \\
221 & $5937 / 5937$ & \\
177 & $3047 / 9396$ & HAT-7 \\
189 & $1506 / 6816$ & HAT-10 \\
236 & $5498 / 5498$ & \\
192 & $4711 / 12137$ & HAT-9 \\
149 & $3210 / 10156$ & HAT-7 \\
247 & $4559 / 4559$ & \\
248 & $5180 / 5180$ & \\
\hline
\end{tabular}

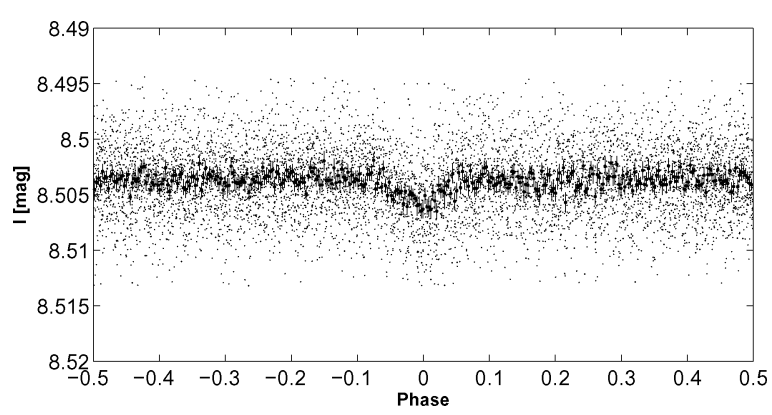

Figure 1. Phase folded light curve of HTR177-004, showing a transit-like shape with a 3 mmag depth. This target was identified as a blend by follow-up observations. The binned light curve is overplotted, in bold. The period is 0.57 days and the light curve contains 7350 measurements.

recent spectroscopic follow-up observations identified this candidate as a false positive, it demonstrates the capability of HATNet-WHAT.

Preliminary spectroscopic follow-up of most of the HATNet-WHAT candidates is done with the CfA Digital Speedometer (DS, Latham 1992), where obvious false positives are identified. Candidates which pass the DS screening are followed up with SOPHIE at the OHP 1.93-m telescope (Bouchy et al. 2006).

WHAT has participated in the discovery of the transiting planet HD147506b, also known as HAT-P-2b (Bakos et al. 2007). The host star was observed by 4 instruments, resulting in 26461 individual measurements. The large amount of measurements and the spread of instruments around the globe enabled the detection of this planet, with a relatively long period of 5.63 days and shallow transit of 5 mmag.

\section{References}

Bakos et al. 2002, PASP, 114, 974

Bakos et al. 2004, PASP, 116, 266

Bakos et al. 2007, ApJ, 670, 826

Bouchy \& the SOPHIE team 2006, Tenth Anniversary of 51 Peg-b, 319

Latham 1992, IAU Colloq. 135, Complementary Approaches to Double and Multiple Star Research, 110 